\section{Fatores associados ao consumo de frutas, legumes e verduras em adultos de uma cidade no Sul do Brasil}

\author{
Factors associated with fruit and vegetable \\ intake among adults in a southern \\ Brazilian city
}

\footnotetext{
${ }^{1}$ Faculdade de Nutrição, Universidade Federal de Pelotas, Pelotas, Brasil. 2 Escola Superior de Educação Física, Universidade Federal de Pelotas, Pelotas, Brasil. 3 Faculdade Atlântico Sul de Pelotas, Pelotas, Brasil. 4 Programa de Pós-graduação em Epidemiologia, Universidade Federal de Pelotas, Pelotas, Brasil.

Correspondência M. B. Neutzling Departamento de Nutrição, Faculdade de Nutrição, Universidade Federal de Pelotas.

Campus Universitário $s / n$, Pelotas, RS

96010-900, Brasil.

mneutzling@terra.com.br
}

\begin{abstract}
The study aimed to describe the frequency of fruit and vegetable intake among adults (20-69 years of age) and to identify associated factors. This population-based study in 2006 included 972 adults in Pelotas, Southern Brazil. Consumption of fruits and vegetables was evaluated with three questions on habitual food intake during the year prior to the interview. The outcome variable was regular consumption of fruits and vegetables. Only one in five adults (20.9\%) reported consuming fruits and vegetables regularly. Female gender, age 60 years or older, higher socioeconomic status, former smoking, and physical activity were associated with the outcome variable. According to the results, fruit and vegetable intake among adults fails to meet current Ministry of Health recommendations, particularly among male, young, poor, and physically inactive adults. Interventions to encourage healthy diet are urgently needed.
\end{abstract}

Diet Surveys; Food Consumption; Food Habits; Adult

\author{
Marilda Borges Neutzling 1 \\ Airton José Rombaldi 2 \\ Mario Renato Azevedo 3 \\ Pedro C. Hallal 2,4
}

\section{Introdução}

O consumo de frutas, legumes e verduras tem sido associado à diminuição do risco de mortalidade 1 e redução da ocorrência de doenças crônicas, tais como as doenças cardiovasculares 2 , derrames 3 e alguns tipos de câncer ${ }^{4}$. Recente publicação da Organização Mundial da Saúde (OMS) 5 afirma que existem evidências convincentes de que o consumo de frutas, legumes e verduras também diminui o risco de diabetes e obesidade. Tal publicação ${ }^{5}$ recomenda um consumo mínimo de pelo menos cinco porções diárias de frutas, legumes e verduras, o que equivale a $400 \mathrm{~g}$ ou mais por dia.

$\mathrm{O}$ aumento na ingestão de frutas, legumes e verduras pela população em geral tem sido uma prioridade das políticas públicas de saúde em muitos países 5,6. Identificar os fatores associados ao consumo de frutas, legumes e verduras pode ser o primeiro passo para orientar os planejadores de saúde em direção às populações de maior risco. De acordo com estudos prévios 7,8, fatores sócio-econômicos como nível educacional e renda familiar são importantes determinantes da baixa ingestão de frutas, legumes e verduras. Em comparação a outros países, como os Estados Unidos e países europeus 7,8,9, existem poucos dados no Brasil com relação à ingestão de frutas, legumes e verduras e fatores associados ao seu consumo. A última pesquisa de orçamentos familiares no Brasil constatou a insuficiência no 
consumo de frutas, legumes e verduras na dieta dos brasileiros em todos os segmentos populacionais 10,11. Resultados da Pesquisa Mundial de Saúde ${ }^{11}$ revelaram que o consumo diário de frutas, legumes e verduras por adultos brasileiros foi referido por apenas $1 / 5$ dos entrevistados, sendo o consumo maior à medida que aumentava a idade, em indivíduos com maior escolaridade e maior número de bens no domicílio 11 . Estudo realizado em 2003 por Figueiredo et al. 12 em São Paulo, Brasil, concluiu que o consumo de frutas, legumes e verduras da população adulta residente em São Paulo foi maior entre as mulheres, sendo influenciado pela idade, escolaridade e dieta.

O objetivo do presente estudo foi descrever a freqüência do consumo de frutas, legumes e verduras por adultos de 20 a 69 anos, residentes na cidade de Pelotas, Rio Grande do Sul, Brasil, e analisar os fatores associados a tal consumo.

\section{Métodos}

Foi conduzido um estudo transversal de base populacional na cidade de Pelotas, no segundo semestre de 2006. Esta cidade está localizada no extremo sul do Estado do Rio Grande do Sul e possui cerca de 340 mil habitantes. O Instituto Brasileiro de Geografia e Estatística (IBGE) divide a cidade em 408 setores censitários urbanos domiciliares, os quais são ordenados no formato "espiral", do centro para os bairros. Dos 404 setores que contêm domicílios, foram sorteados, sistematicamente, 40 para serem incluídos no estudo. Em cada setor sorteado, foi escolhido aleatoriamente um ponto de partida para a escolha das residências a serem visitadas. A primeira casa foi incluída e as próximas foram selecionadas de forma sistemática, respeitando-se um pulo de sete domicílios, até atingir 15 moradias em cada setor. No total, foram selecionados 600 domicílios para participar do estudo. Nesses, todos os moradores com idade entre 20 e 69 anos foram incluídos na amostra, exceto aqueles com incapacidade mental ou física severa que os impedisse de responder ao questionário.

O desfecho - consumo de frutas, legumes e verduras - foi estabelecido por meio das perguntas: (1) em geral desde <mês> do ano passado, quantas vezes tu comeste frutas? (sem contar sucos); (2) em geral desde <mês> do ano passado, quantas vezes tu comeste salada verde?; e (3) em geral desde <mês> do ano passado, quantas vezes tu comeste outros vegetais?. O tempo de consumo pregresso investigado em relação ao momento da entrevista foi de 12 meses. As opções de resposta eram: (a) 1 vez ou menos por mês; (b) 2 a 3 vezes por mês; (c) 1 a 2 vezes por semana; (d) 3 a 4 vezes por semana; (e) 5 ou + vezes por semana. $\mathrm{O}$ desfecho foi dicotomizado em consumo regular de frutas, legumes e verduras (cinco ou mais vezes por semana - sim ou não). Dessa forma foi então criada uma variável combinando esses três consumos (frutas, legumes e verduras). O manual de instruções dos entrevistadores continha exemplos de salada verde (alface, rúcula, agrião, couve) e de outros vegetais (cenoura, beterraba, chuchu, abóbora, vagem, couve-flor). Ficou definido também que salada de batata e salada de mandioca não entrariam na denominação "outros vegetais".

Características demográficas, sócio-econômicas e de saúde foram avaliadas pela aplicação de um questionário padronizado, contendo 100 questões. A variável cor da pele foi observada pelos entrevistadores e o nível sócio-econômico determinado segundo classificação da Associação Brasileira de Empresas de Pesquisa 13. O nível de atividade física foi avaliado pela seção de lazer da versão longa do IPAQ (Questionário Internacional de Atividades Físicas) 14. Utilizou-se um ponto de corte de 150 minutos por semana para classificar os sujeitos como ativos ou sedentários 14 . O estado nutricional foi determinado pelo índice de massa corporal (IMC), calculado pelo peso e altura referidos e classificado segundo critérios da OMS 15.

Os entrevistadores selecionados foram de ambos os sexos, com idade mínima de 18 anos e pelo menos ensino médio completo, tendo participado de um treinamento teórico-prático de 20 horas. Os questionários foram revisados por supervisores (acadêmicos dos cursos de Educação Física e Fisioterapia), que estavam em contato permanente com os entrevistadores durante o trabalho de campo. Os mesmos realizaram controle de qualidade, mediante re-visitas a $25 \%$ da amostra. Os dados obtidos pelos questionários foram duplamente digitados no programa Epi Info (Centers for Disease Control and Prevention, Atlanta, Estados Unidos) e as análises conduzidas com o programa estatístico Stata 9.0 (Stata Corp., College Station, Estados Unidos).

$\mathrm{Na}$ análise bruta, foi verificada a relação entre o desfecho e as variáveis sexo, idade, cor da pele, nível econômico, escolaridade, tabagismo, atividade física no lazer e estado nutricional. Para isso, foram utilizados os testes de qui-quadrado para heterogeneidade e tendência linear. A análise multivariável foi realizada por meio de regressão de Poisson, com variância robusta. Todas as análises levaram em consideração o efeito do delineamento amostral, pelo grupo de comandos "svy" do Stata. O nível de significância adotado foi de $5 \%$. 
O projeto foi aprovado pelo Comitê de Ética em Pesquisa da Escola Superior de Educação Física da Universidade Federal de Pelotas e os dados foram coletados após consentimento informado dos sujeitos.

\section{Resultados}

Com base em 514 domicílios que tinham moradores na faixa etária estabelecida, o número de indivíduos elegíveis foi 1.062. Destes, 972 responderam ao questionário, fato que representa uma taxa de não respondentes de 8,5\%. A variável independente com maior número de valores ignorados foi o IMC, com 87 indivíduos não sabendo informar seus valores de peso e/ou altura.

A Tabela 1 mostra que mais da metade da amostra (57\%) era do sexo feminino, aproximadamente $1 / 4$ dos indivíduos $(26,1 \%)$ tinha entre 20 e 29 anos de idade. O nível sócio-econômico mais freqüente foi a classe C $(41,5 \%)$ e grande parte dos indivíduos (82\%) eram brancos e sedentários no lazer (69,8\%). Verificou-se que cerca de metade dos adultos nunca fumou $(49,4 \%) \mathrm{e}$ tinha excesso de peso $(51,3 \%)$.

Na Tabela 2, observa-se que somente 1/5 dos entrevistados $(20,9 \%)$ relatou consumo regular de frutas, legumes e verduras. Essa freqüência de consumo foi substancialmente maior nas mulheres (26,9\%) do que nos homens (12,9\%). Menos da metade dos indivíduos referiu consumo regular de frutas $(43,5 \%)$, legumes $(36,4 \%)$ e verduras (41,5\%), e entre indivíduos do sexo masculino a freqüência de consumo foi significativamente menor.

A Tabela 3 apresenta a freqüência de consumo regular de frutas, legumes e verduras, assim como as razões de prevalência segundo as variáveis independentes. Não foram observadas diferenças estatisticamente significantes quanto à cor da pele e estado nutricional. Nesta análise bruta, indivíduos do sexo feminino, com 60 anos ou mais, das classes A e B, ex-fumantes e não sedentários apresentaram maior prevalência de consumo de frutas, legumes e verduras. Tanto o maior nível sócio-econômico quanto possuir mais idade mostraram-se diretamente associados com a freqüência de consumo de frutas, legumes e verduras. Após ajuste para idade (dados não apresentados na tabela), a associação entre tabagismo e consumo de frutas, legumes e verduras perdeu a significância estatística $(p=0,18)$ e as demais associações não se modificaram.

As Tabelas 4 e 5 mostram os resultados para os sexos feminino e masculino separadamente. Observa-se que o aumento da idade e a prática de atividade física no lazer estiveram positiva- mente associados à maior freqüência de consumo de frutas, legumes e verduras em ambos os sexos. No sexo feminino, observaram-se ainda associações entre nível sócio-econômico e tabagismo com consumo de frutas, legumes e verduras. As mulheres do estrato superior de nível sócio-econômico tiveram 2,25 vezes maior chance (IC95\%: 1,38-3,65) de consumir regularmente frutas, legumes e verduras, quando comparadas com aquelas do estrato inferior de nível sócioeconômico. Mulheres ex-fumantes tiveram $66 \%$ (IC95\%: 1,11-2,49) mais chance de consumo regular de frutas, legumes e verduras do que as fumantes atuais.

\section{Discussão}

Um aspecto a ser destacado nesse estudo é que a amostra pode ser considerada representativa dos adultos de 20 a 69 anos de idade residentes em Pelotas, tendo em vista o alto percentual de indivíduos entrevistados e o baixo índice de perdas e recusas. Algumas limitações também precisam ser consideradas. As diferenças entre os métodos para avaliar dieta, definir e categorizar a freqüência de consumo de frutas, legumes e verduras prejudicam a comparação com outros estudos sobre consumo populacional. Existe também a possibilidade do viés de causalidade reversa na associação entre freqüência de consumo de frutas, legumes e verduras e estado nutricional, visto que pessoas obesas podem modificar o consumo como conseqüência de seu estado nutricional. Além disso, deve-se considerar a possibilidade da ocorrência de um erro de recordatório, uma vez que o tempo de consumo pregresso investigado em relação ao momento da entrevista foi de 12 meses.

Nosso estudo mostrou que apenas uma minoria $(20,9 \%)$ dos indivíduos adultos em Pelotas consumia frutas, legumes e verduras regularmente. Essa freqüência de consumo é bem inferior àquelas relatadas em estudos internacionais. Estudo de Estaquio et al. 16, com adultos franceses de ambos os sexos, constatou freqüência de consumo diário de 5 ou mais porções de frutas, legumes e verduras em cerca de $50 \%$ dos homens e mulheres adultas. Nos Estados Unidos, em 2005, o Centers for Disease Control and Prevention (CDC) 17 verificou que $32 \%$ e $27,2 \%$ da população norte-americana consumiam, respectivamente, frutas e legumes e verduras três ou mais vezes por dia. Destaque-se que o desfecho de nosso estudo considerou a freqüência de consumo regular (cinco ou mais vezes por semana) enquanto o de Estaquio et al. 16 e o CDC 17 verificou o número de porções por dia. Por outro 
Descrição da amostra em termos de variáveis sócio-demográficas, comportamentais e de saúde. Pelotas, Rio Grande do Sul, Brasil, $2006(\mathrm{~N}=972)$.

\begin{tabular}{|c|c|c|}
\hline Variáveis & $\mathrm{n}$ & $\%$ \\
\hline \multicolumn{3}{|l|}{ Sexo $[n=972]$} \\
\hline Homens & 418 & 43,0 \\
\hline Mulheres & 554 & 57,0 \\
\hline \multicolumn{3}{|c|}{ Idade (anos) [n = 972] } \\
\hline $20-29$ & 254 & 26,1 \\
\hline $30-39$ & 195 & 20,1 \\
\hline $40-49$ & 247 & 25,4 \\
\hline $50-59$ & 173 & 17,8 \\
\hline $60-69$ & 103 & 10,6 \\
\hline \multicolumn{3}{|c|}{ Nível sócio-econômico * (classes) [n = 952] } \\
\hline$A$ e B & 382 & 40,1 \\
\hline C & 395 & 41,5 \\
\hline $\mathrm{De} E$ & 175 & 18,4 \\
\hline \multicolumn{3}{|c|}{ Cor da pele $[\mathrm{n}=972]$} \\
\hline Branca & 797 & 82,0 \\
\hline Não branca & 175 & 18,0 \\
\hline \multicolumn{3}{|c|}{ Tabagismo [n = 972] } \\
\hline Ex-fumante & 216 & 22,2 \\
\hline Fumante atual & 276 & 28,4 \\
\hline Nunca fumou & 480 & 49,4 \\
\hline \multicolumn{3}{|c|}{ Sedentarismo no lazer $[\mathrm{n}=968]$} \\
\hline Sim & 676 & 69,8 \\
\hline Não & 292 & 30,2 \\
\hline \multicolumn{3}{|c|}{ Estado nutricional $[n=885]$} \\
\hline Obesidade & 133 & 15,0 \\
\hline Sobrepeso & 321 & 36,3 \\
\hline Eutrófico & 431 & 48,7 \\
\hline
\end{tabular}

* Associação Brasileira de Empresas de Pesquisa 13.

Nota: o maior número de valores ignorados $(n=87)$ foi para a variável estado nutricional.

lado, estudos brasileiros 11,12,18 encontraram resultados semelhantes aos nossos. Jaime \& Monteiro 11, analisando dados da Pesquisa Mundial de Saúde no Brasil, observaram que a freqüência de consumo diário de frutas, legumes e verduras ocorria em apenas $20,4 \%$ dos entrevistados e que a ingestão adequada (cinco ou mais porções de frutas, legumes e verduras ao dia) era relatada por apenas $13,5 \%$ dos adultos. Figueiredo et al. 12 , em 2008, estudando separadamente o consumo diário de frutas, legumes e verduras de adultos paulistanos, verificaram que $43,9 \%$ dos adultos consumiam frutas diariamente, $46,8 \%$ verduras e somente $18,1 \%$ consumiam legumes, resulta- dos consistentes com nossos achados. Estudos monitorando fatores de risco para doenças não transmissíveis com base em entrevistas telefônicas encontraram freqüência de consumo regular de frutas e hortaliças em $47 \%$ dos entrevistados em Goiânia 18 e 15,6\% em Belém 19. Resultados do Vigitel 2007 (Vigilância de Fatores de Risco e Proteção para Doenças Crônicas por Inquérito Telefônico) 20 indicam que a freqüência de adultos brasileiros consumindo regularmente frutas e hortaliças foi modesta na maioria das cidades estudadas, variando entre 16,8\% em Rio Branco (Acre) e $40 \%$ em Porto Alegre (Rio Grande do Sul, $30,1 \%$ no sexo masculino e $48,1 \%$ no sexo feminino). 
Descrição da amostra segundo variáveis de consumo alimentar e sexo. Pelotas, Rio Grande do Sul, Brasil, 2006 (N = 972 ).

\begin{tabular}{|c|c|c|c|c|c|c|}
\hline \multirow[t]{2}{*}{ Variáveis } & \multicolumn{2}{|c|}{ Total } & \multicolumn{2}{|c|}{ Sexo feminino } & \multicolumn{2}{|c|}{ Sexo masculino } \\
\hline & $\%$ & IC95\% & $\%$ & IC95\% & $\%$ & IC95\% \\
\hline Consumo regular de frutas $[n=960]$ & 43,5 & $0,40-0,47$ & 52,5 & $0,49-0,56$ & 31,7 & $0,29-0,35$ \\
\hline Consumo regular de outros vegetais (legumes) $[n=961]$ & 36,4 & $0,33-0,40$ & 42,3 & $0,39-0,46$ & 28,6 & $0,26-0,32$ \\
\hline Consumo regular de salada verde (verduras) [n =962] & 45,1 & $0,42-0,48$ & 50,6 & $0,47-0,54$ & 37,8 & $0,35-0,41$ \\
\hline Consumo regular de frutas, legumes e verduras [ $n=956$ ] & 20,9 & $0,18-0,24$ & 26,9 & $024-0,30$ & 12,9 & $0,11-0,15$ \\
\hline
\end{tabular}

IC95\%: intervalo de 95\% de confiança.

Nota: o maior número de valores ignorados $(n=16)$ foi para a variável consumo regular de frutas, legumes e verduras.

Tabela 3

Associação das variáveis demográficas, sócio-econômicas, comportamentais e de saúde com o consumo regular de frutas, legumes e verduras em Pelotas, Rio Grande do Sul, Brasil, 2006 ( $\mathrm{N}=956)$.

\begin{tabular}{|c|c|c|c|c|}
\hline \multirow[t]{2}{*}{ Variáveis } & \multicolumn{2}{|c|}{ Consumo regular de frutas, legumes e verduras } & \multirow{2}{*}{$\begin{array}{l}\text { Razões de prevalência } \\
\text { (IC95\%) }\end{array}$} & \multirow[t]{2}{*}{ Valor de $p$} \\
\hline & $\mathbf{n}$ & $\%$ & & \\
\hline Sexo & & & & $<0,001$ * \\
\hline Masculino & 53 & 12,9 & 1,00 & \\
\hline Feminino & 147 & 26,9 & $2,08(1,56-2,77)$ & \\
\hline Idade (anos) & & & & $<0,001$ * \\
\hline $20-29$ & 35 & 13,9 & 1,00 & \\
\hline $30-39$ & 30 & 15,8 & $1,14(0,72-1,78)$ & \\
\hline $40-49$ & 56 & 22,9 & $1,65(1,12-2,42)$ & \\
\hline $50-59$ & 48 & 27,9 & $2,00(1,26-2,97)$ & \\
\hline 60 ou mais & 31 & 32,0 & $2,30(1,51-3,51)$ & \\
\hline Nível sócio-econômico (classes) ** & & & & $<0,001$ * \\
\hline$A$ e $B$ & 102 & 27,0 & $2,12(1,39-234)$ & \\
\hline C & 74 & 18,8 & $1,48(0,95-2,30)$ & \\
\hline$D$ e $E$ & 22 & 12,7 & 1,00 & \\
\hline Cor da pele & & & & $0,09 * \star \star$ \\
\hline Branca & 172 & 22,0 & $1,37(0,95-1,97)$ & \\
\hline Não branca & 28 & 16,1 & 1,00 & \\
\hline Tabagismo & & & & 0,03 * \\
\hline Fumante atual & 47 & 17,3 & 1,00 & \\
\hline Nunca fumou & 96 & 20,3 & $1,18(0,86-1,61)$ & \\
\hline Ex-fumante & 57 & 26,9 & $1,56(1,11-2,19)$ & \\
\hline Sedentarismo no lazer & & & & 0,002 * \\
\hline Sim & 122 & 18,3 & 1,00 & \\
\hline Não & 78 & 27,3 & $1,49(1,16-1,91)$ & \\
\hline
\end{tabular}

(continua) 
Tabela 3 (continuação)

\begin{tabular}{lccc}
\hline Variáveis & $\begin{array}{c}\text { Consumo regular de frutas, legumes e verduras } \\
\%\end{array}$ & $\begin{array}{c}\text { Razões de prevalência } \\
\text { (IC95\%) }\end{array}$ & Valor de $\mathbf{p}$ \\
\hline Estado nutricional & $\mathbf{n}$ & & \\
Obesidade & 31 & 24,0 & $0,75 * * *$ \\
Sobrepeso & 69 & 21,8 & $1,15(0,80-1,65)$ \\
Eutrófico & 89 & 20,9 & $1,04(0,79-1,38)$ \\
\hline
\end{tabular}

IC95\%: intervalo de 95\% de confiança.

* Teste do qui-quadrado para tendência linear;

** Associação Brasileira de Empresas de Pesquisa 13;

*** Teste do qui-quadrado para heterogeneidade.

Associação das variáveis demográficas, sócio-econômicas, comportamentais e de saúde com o consumo regular de frutas, legumes e verduras em Pelotas, Rio Grande do Sul, Brasil, 2006 - sexo masculino ( $n=410)$.

\begin{tabular}{|c|c|c|c|c|}
\hline \multirow[t]{2}{*}{ Variáveis } & \multicolumn{2}{|c|}{ Consumo regular de frutas, legumes e verduras } & \multirow{2}{*}{$\begin{array}{l}\text { Razões de prevalência } \\
\text { (IC95\%) }\end{array}$} & \multirow[t]{2}{*}{ Valor de $\mathrm{p}$} \\
\hline & $\mathrm{n}$ & $\%$ & & \\
\hline Idade (anos) & & & & 0,04 * \\
\hline $20-29$ & 11 & 9,2 & 1,00 & \\
\hline $30-39$ & 4 & 5,3 & $0,57(0,19-1,73)$ & \\
\hline $40-49$ & 15 & 15,3 & $1,66(0,80-3,44)$ & \\
\hline $50-59$ & 14 & 18,4 & $1,99(0,95-4,16)$ & \\
\hline 60 ou mais & 9 & 22,0 & $2,37(1,06-5,32)$ & \\
\hline Nível sócio-econômico (classes) ** & & & & 0,14 *** \\
\hline$A$ e $B$ & 28 & 16,5 & $1,98(0,85-4,57)$ & \\
\hline C & 17 & 10,6 & $1,27(0,52-3,08)$ & \\
\hline$D$ e $E$ & 6 & 8,3 & 1,00 & \\
\hline Cor da pele & & & & 0,28 *** \\
\hline Branca & 47 & 13,7 & $1,56(0,69-3,50)$ & \\
\hline Não branca & 6 & 8,8 & 1,00 & \\
\hline Tabagismo & & & & $0,23 * * *$ \\
\hline Fumante atual & 17 & 12,8 & 1,00 & \\
\hline Nunca fumou & 18 & 10,3 & $0,81(0,43-1,51)$ & \\
\hline Ex-fumante & 18 & 17,5 & $1,37(0,74-2,52)$ & \\
\hline Sedentarismo no lazer & & & & 0,03 *** \\
\hline Sim & 26 & 10,2 & 1,00 & \\
\hline Não & 27 & 17,6 & $1,73(1,05-2,85)$ & \\
\hline Estado nutricional & & & & 0,76 * \\
\hline Obesidade & 7 & 13,0 & $1,09(0,49-242)$ & \\
\hline Sobrepeso & 24 & 14,6 & $1,22(0,71-2,12)$ & \\
\hline Eutrófico & 21 & 11,9 & 1,00 & \\
\hline
\end{tabular}

IC95\%: intervalo de 95\% de confiança.

* Teste do qui-quadrado para tendência linear;

** Associação Brasileira de Empresas de Pesquisa 13;

*** Teste do qui-quadrado para heterogeneidade. 
Associação das variáveis demográficas, sócio-econômicas, comportamentais e de saúde com o consumo regular de frutas, legumes e verduras em Pelotas, Rio Grande do Sul, Brasil, 2006 - sexo feminino ( $n=546$ ).

\begin{tabular}{|c|c|c|c|c|}
\hline \multirow[t]{2}{*}{ Variáveis } & \multicolumn{2}{|c|}{ Consumo regular de frutas, legumes e verduras } & \multirow{2}{*}{$\begin{array}{l}\text { Razões de prevalência } \\
\text { (IC95\%) }\end{array}$} & \multirow[t]{2}{*}{ Valor de $\mathrm{p}$} \\
\hline & $n$ & $\%$ & & \\
\hline Idade (anos) & & & & 0,007 * \\
\hline $20-29$ & 24 & 18,0 & 1,00 & \\
\hline $30-39$ & 26 & 22,8 & $1,26(0,77-2,07)$ & \\
\hline $40-49$ & 41 & 27,9 & $1,55(0,99-2,41)$ & \\
\hline $50-59$ & 34 & 35,4 & $1,96(2,25-3,08)$ & \\
\hline 60 ou mais & 22 & 39,3 & $2,18(1,33-3,54)$ & \\
\hline Nível sócio-econômico (classes) ** & & & & 0,001 * \\
\hline$A$ e $B$ & 74 & 35,6 & $2,25(1,38-3,65)$ & \\
\hline C & 57 & 24,6 & $1,55(0,94-2,57)$ & \\
\hline $\mathrm{DeE}$ & 16 & 15,8 & 1,00 & \\
\hline Cor da pele & & & & $0,12^{\star \star \star}$ \\
\hline Branca & 125 & 28,4 & $1,37(0,92-2,04)$ & \\
\hline Não branca & 22 & 20,8 & 1,00 & \\
\hline Tabagismo & & & & $0,04 * \star \star$ \\
\hline Fumante atual & 30 & 21,6 & 1,00 & \\
\hline Nunca fumou & 78 & 26,2 & $1,21(0,84-1,76)$ & \\
\hline Ex-fumante & 39 & 35,8 & $1,66(1,11-2,49)$ & \\
\hline Sedentarismo no lazer & & & & $<0,001$ * \\
\hline Sim & 96 & 23,3 & 1,00 & \\
\hline Não & 51 & 38,3 & $1,65(1,25-2,17)$ & \\
\hline Estado nutricional & & & & 0,70 * \\
\hline Obesidade & 24 & 32,0 & $1,18(0,80-1,73)$ & \\
\hline Sobrepeso & 45 & 29,4 & $1,08(0,79-1,49)$ & \\
\hline Eutrófico & 68 & 27,2 & 1,00 & \\
\hline
\end{tabular}

IC95\%: intervalo de 95\% de confiança.

* Teste do qui-quadrado para tendência linear;

** Associação Brasileira de Empresas de Pesquisa 13;

$\star \star \star$ Teste do qui-quadrado para heterogeneidade.

No presente estudo, as freqüências de consumo de frutas, legumes e verduras foram maiores entre as mulheres, em indivíduos mais velhos e entre aqueles que possuíam maior nível sócioeconômico. Resultados similares foram encontrados em vários outros estudos 12,16,17,20. Na Europa, Estaquio et al. 16 encontraram maior consumo de frutas, legumes e verduras em indivíduos mais velhos, de maior escolaridade e maior nível econômico. Nos Estados Unidos, em 2005, o CDC 17 constatou freqüências de consumo de legumes e verduras mais elevadas em mulheres, em indivíduos com mais de 65 anos e com maior nível de escolaridade. No Brasil, Figueiredo et al. 12, em estudo transversal realizado em São Paulo, verificaram que mulheres mais velhas e de maior escolaridade consumiam frutas, legumes e verduras com maior freqüência.

Recentes estudos de revisão 8,21 têm analisado e discutido os determinantes ambientais e sócio-econômicos do consumo de frutas, legumes e verduras. Damon \& Drewnowski 21 buscam explicar os mecanismos causais da associação entre nível sócio-econômico e qualidade da dieta. Concluem que muito além do conhecimento nutricional ou motivação para o consumo está o custo dos alimentos. Recente estudo inglês 22 demonstrou que o fornecimento de cupons para 
a compra de frutas e verduras foi uma medida efetiva para promover o aumento do consumo desses alimentos entre mulheres de baixa renda. No Brasil, em 2007, Claro et al. 23, analisando dados da Pesquisa de Orçamentos Familiares no Município de São Paulo, concluíram que 1\% de diminuição de preço das frutas, legumes e verduras aumentaria em $0,2 \%$ sua participação no total calórico. Por outro lado, Pomerleau et al. 24, em uma revisão sistemática sobre intervenções para aumentar o consumo de frutas, legumes e verduras em países desenvolvidos, verificaram que o aconselhamento nutricional em nível populacional em períodos $\geq 3$ meses foi capaz de aumentar o consumo em 0,1 a 1,4 porções de frutas, legumes e verduras ao dia. No Brasil, em 2004, ensaio comunitário realizado em um bairro pobre do Município de São Paulo, buscando aumentar a participação de frutas e hortaliças na alimentação familiar, concluiu que o grupointervenção apresentou um acréscimo de 2,9 pontos percentuais (IC95\%: 0,32-5,39) no total de calorias provenientes de frutas e hortaliças 25 . Considerando que hábitos alimentares estão inseridos em estruturas culturais, econômicas e políticas, entende-se que é necessário haver maior ênfase na promoção de políticas dirigidas aos determinantes do consumo de frutas, legumes e verduras, bem como em ações específicas de educação nutricional que visem ao estímulo do consumo desses alimentos.

No que se refere à associação entre tabagismo e consumo de frutas, legumes e verduras, verificou-se que entre ex-fumantes a freqüência de consumo desses alimentos é significativamente maior. Resultados similares foram encontrados nos estudos de Elizondo et al. 26 (Espanha, 2005) e de Birkett 27 (Canadá, 1999). Corroborando nossos achados, Elizondo et al. 26 concluem que os ex-fumantes são a categoria que apresenta padrão de consumo alimentar mais saudável. Birkett 27 verificou que os fumantes consumiam substancialmente menos frutas e sucos de fruta que os não-fumantes e que os fumantes pesados tinham quatro a seis vezes mais chance de ingerir menos frutas que o recomendado. Resultados similares, mas menos marcantes, foram encontrados com relação ao consumo de legumes e verduras.

A análise da relação entre sedentarismo no lazer e o consumo de frutas, legumes e verduras revelou que indivíduos menos ativos consomem menos frutas, legumes e verduras. Outros estudos 16,28,29 encontraram resultados semelhantes. Camões \& Lopes 28, em 2008, estudando adultos portugueses de ambos os sexos, observaram que maiores níveis de atividade física estiveram associados com dietas mais saudáveis. Estaquio et al. 16, em 2008, na França, verificaram que mulheres mais ativas foram aquelas com maior consumo de frutas, legumes e verduras, embora o critério para definição de inatividade física tenha considerado também a prática de atividade física nos domínios do trabalho e do deslocamento, além do lazer. Em 2003, num amplo estudo de coorte na Espanha, Tormo et al. 29 constataram um aumento de $15,9 \%$ no consumo de vegetais e $6,7 \%$ no consumo de frutas quando comparados os níveis mais baixos e mais altos de atividade física no lazer.

Com base nos resultados obtidos, é possível concluir que a freqüência do consumo de frutas, legumes e verduras na população adulta residente no Município de Pelotas está muito aquém das recomendações atuais do Ministério da Saúde do Brasil (5 porções de frutas, legumes e verduras ao dia), em especial entre os homens mais jovens, de menor nível sócio-econômico e que não praticam atividade física no lazer. Políticas públicas dirigidas aos determinantes do consumo de frutas, legumes e verduras são urgentes e necessárias. Isso pode incluir subsídios à produção desses alimentos, ações de incentivo à maior freqüência de consumo de frutas, legumes e verduras, bem como a prática de atividade física. 


\section{Resumo}

O objetivo deste estudo foi descrever a freqüência de consumo de frutas, legumes e verduras por adultos de 20 a 69 anos de Pelotas, Rio Grande do Sul, Brasil, e analisar fatores associados. Foi realizado em 2006 um estudo transversal de base populacional, incluindo 972 adultos. A freqüência do consumo de frutas, legumes e verduras foi medida por meio de três perguntas referentes ao consumo habitual destes no ano anterior. O desfecho foi o consumo regular de frutas, legumes $e$ verduras. Cerca de 1/5 da população adulta (20,9\%) consumia regularmente frutas, legumes e verduras. Indivíduos do sexo feminino, com 60 anos ou mais, das classes A e B, ex-fumantes e não sedentários apresentaram maior prevalência de consumo de frutas e legumes e verduras. A freqüência do consumo de frutas, legumes e verduras na população adulta residente no Município de Pelotas está aquém das recomendações atuais do Ministério da Saúde, em especial entre os homens mais jovens, de menor nível sócio-econômico e que não praticam atividade física no lazer. Políticas públicas que estimulem uma alimentação saudável são urgentemente necessárias.

Inquéritos sobre Dieta; Consumo de Alimentos; Hábitos Alimentares; Adulto

\section{Colaboradores}

M. B. Neutzling e A. J. Rombaldi realizaram a revisão de literatura, conduziram as análises, confecção das tabelas, interpretação, escrita dos resultados e discussão. M. R. Azevedo e P. C. Hallal supervisionaram o trabalho de campo e ajudaram na escrita do artigo, em todas as fases.

\section{Referências}

1. Agudo A, Cabrera L, Amiano P, Ardanaz E, Barricarte A, Berenguer T, et al. Fruit and vegetable intakes, dietary antioxidant nutrients, and total mortality in Spanish adults: findings from the Spanish cohort of the European Prospective Investigation into Cancer and Nutrition (EPIC-Spain). Am J Clin Nutr 2007; 85:1634-42.

2. Dauchet L, Amouyel P, Hercberg S, Dallongeville J. Fruit and vegetable consumption and risk of coronary heart disease: a meta-analysis of cohort studies. J Nutr 2006; 136:2588-93.

3. He FJ, Nowson CA, MacGregor GA. Fruit and vegetable consumption and stroke-meta-analysis of cohort studies. Lancet 2006; 367:320-6.

4. Key TJ, Allen NE, Spencer EA, Travis RC. The effect of diet on risk of cancer. Lancet 2002; 360:861-8.

5. World Health Organization. Diet nutrition and the prevention of chronic diseases: report of a joint WHO/FAO Expert Consultation. Geneva: World Health Organization; 2002. (WHO Technical Report Series, 916).
6. Coordenação-Geral da Política de Alimentação e Nutrição, Secretaria de Atenção à Saúde, Ministério da Saúde. Guia alimentar para a população brasileira. Brasília: Ministério da Saúde; 2005.

7. Irala-Estevez JD, Groth M, Johansson L, Oltersdorf U, Prattala R, Martinez-Gonzalez MA. A systematic review of socio-economic differences in food habits in Europe: consumption of fruit and vegetables. Eur J Clin Nutr 2000; 54:706-14.

8. Kamphuis CB, Giskes K, Bruijn GJ, Wendel-Vos W, Brug J, van Lenthe FJ. Environmental determinants of fruit and vegetable consumption among adults-a systematic review. Br J Nutr 2006; 96: 620-35.

9. Roos G, Johansson L, Kasmel A, Klumbiene J, Prattala R. Disparities in vegetable and fruit consumption: European cases from the north to the south. Public Health Nutr 2001; 4:35-43. 
10. Instituto Brasileiro de Geografia e Estatística. Pesquisa de Orçamentos Familiares 2002-2003: análise da disponibilidade domiciliar de alimentos e do estado nutricional no Brasil. Rio de Janeiro: Instituto Brasileiro de Geografia e Estatística; 2004.

11. Jaime PC, Monteiro CA. Fruit and vegetable intake by Brazilian adults, 2003. Cad Saúde Pública 2005; 21 Suppl 1:S19-24.

12. Figueiredo IC, Jaime PC, Monteiro CA. Factors associated with fruit and vegetable intake among adults of the city of Sao Paulo, Southeastern Brazil. Rev Saúde Pública 2008; 42:777-85.

13. Associação Brasileira de Empresas de Pesquisa. Critério de classificação econômica Brasil. http: / / www.abep.org/codigosguias/CCEB2008 Base2006e2007.pdf.2006 (acessado em 25/Fev/ 2009).

14. Craig CL, Marshall AL, Sjostrom M, Bauman AE, Booth ML, Ainsworth BE, et al. International physical activity questionnaire: 12-country reliability and validity. Med Sci Sports Exerc 2003; 35:1381-95.

15. World Health Organization. Physical status: the use and interpretation of anthropometry. Report of a WHO Expert Committee. Geneva: World Health Organization; 1995. (WHO Technical Report Series, 854)

16. Estaquio C, Druesne-Pecollo N, Latino-Martel P, Dauchet L, Hercberg S, Bertrais S. Socioeconomic differences in fruit and vegetable consumption among middle-aged French adults: adherence to the 5 a day recommendation. J Am Diet Assoc 2008; 108:2021-30.

17. Centers for Disease Control and Prevention. Fruit and vegetable consumption among adults - United States, 2005. MMWR Morb Mortal Wkly Rep 2007; 56:213-7.

18. Peixoto MR, Monego ET, Alexandre VP, Souza RG, Moura EC. Monitoramento por entrevistas telefônicas de fatores de risco para doenças crônicas: experiência de Goiânia, Goiás, Brasil. Cad Saúde Pública 2008; 24:1323-33.
19. Moura EC, Dias RM, Reis RC. Determinantes do consumo de frutas, legumes e verduras na população adulta de Belém, Pará, 2005. Nutrire Rev Soc Bras Aliment Nutr 2007; 32:29-40.

20. Ministério da Saúde. Vigitel Brasil 2007. Vigilância de fatores de risco e proteção para doenças crônicas por inquérito telefônico. Brasília: Ministério da Saúde; 2009.

21. Darmon N, Drewnowski A. Does social class predict diet quality? Am J Clin Nutr 2008; 87:1107-17.

22. Burr ML, Trembeth J, Jones KB, Geen J, Lynch LA, Roberts ZE. The effects of dietary advice and vouchers on the intake of fruit and fruit juice by pregnant women in a deprived area: a controlled trial. Public Health Nutr 2007; 10:559-65.

23. Claro RM, Carmo HC, Machado FM, Monteiro CA. Renda, preço dos alimentos e participação de frutas e hortaliças na dieta. Rev Saúde Pública 2007; 41:557-64.

24. Pomerleau J, Lock K, Knai C, McKee M. Interventions designed to increase adult fruit and vegetable intake can be effective: a systematic review of the literature. J Nutr 2005; 135:2486-95.

25. Jaime PC, Machado FM, Westphal MF, Monteiro CA. Educação nutricional e consumo de frutas e hortaliças: ensaio comunitário controlado. Rev Saúde Pública 2007; 41:154-7.

26. Elizondo JJ, Guillen F, Aguinaga I. Diferencias en el consumo de alimentos y nutrientes según el hábito tabáquico. An Sist Sanit Navar 2006; 29:37-46.

27. Birkett NJ. Intake of fruits and vegetables in smokers. Public Health Nutr 1999; 2:217-22.

28. Camoes M, Lopes C. Dietary intake and different types of physical activity: full-day energy expenditure, occupational and leisure-time. Public Health Nutr 2008; 11:841-8.

29. Tormo MJ, Navarro C, Chirlaque MD, Barber X, Argilaga S, Agudo A, et al. Physical sports activity during leisure time and dietary intake of foods and nutrients in a large Spanish cohort. Int J Sport Nutr Exerc Metab 2003; 13:47-64.

Recebido em 07/Mai/2009

Versão final reapresentada em 06/Jul/2009

Aprovado em 29/Jul/2009 